“ (C) 2017 IEEE. Personal use of this material is permitted. Permission from IEEE must be obtained for all other uses, in any current or future media, including

reprinting/republishing this material for advertising or promotional purposes, creating new collective works, for resale or redistribution to servers or lists, or reuse of any copyrighted component of this work in other works." 


\title{
WarnFi: Non-Invasive WiFi-based Abnormal Activity Sensing Using Non-parametric Model
}

\author{
Na Pang*†, Dali Zhu*†, Gang $\mathrm{Li}^{\ddagger}$, Shaowu Liu ${ }^{\S}$ \\ * Institute of Information Engineering, Chinese Academy of Sciences, Beijing, China \\ ${ }^{\dagger}$ School of Cyber Security, University of Chinese Academy of Sciences, Beijing, China \\ ${ }^{\ddagger}$ School of Information Technology, Deakin University, Geelong, Australia \\ $\S$ Advanced Analytics Institute, University of Technology Sydney, Sydney, Australia \\ Email: \{pangna, zhudali\}@iie.ac.cn, gang.li@ deakin.edu.au, shaowu.liu@uts.edu.au
}

\begin{abstract}
Abnormal activity sensing has attracted increasing research attention in military surveillance, patient monitoring, and health care of children and elderly, etc. Researchers have exploited the characteristics of wireless signals to sense "keystrokes" and "human talks", relieving the privacy invasion concern caused by mounting the surveillance cameras or wearing the smart devices. However, existing technologies usually require some specialized hardware, and can only sense a fixed set of pre-defined activities through a supervised learning from those wireless signals patterns. In this paper, we propose WarnFi, a non-invasive abnormal activity sensing system with only two commodity off-the-shelf (COTS) WiFi devices. The intuition of WarnFi is that whenever the human body occludes the wireless signal transmitting from the access point to the receiver, the time-series of Channel State Information (CSI) will experience a unique variation. By using a non-parametric model, WarnFi can dynamically cluster the human body activities for abnormal sensing. Extensive experiments in various scenarios demonstrate the satisfactory performance of WarnFi.
\end{abstract}

\section{INTRODUCTION}

Abnormal activity sensing, the ability of finding "rare and different" activities that do not conform to expected patterns, has become an important task in many applications, such as military surveillance, health care, patient monitoring, etc. [1]. For example, if soldiers' abnormal activity can be sensed automatically, immediate alerts would be possible in case of emergency or injury. Traditional vision based systems, such as Xbox Kinect [2] and HON4D [3], require the line-of-sight (LOS) with adequate lighting, and bring the new concern in privacy disclosure. Wearable sensor based systems cause inconvenience in occasions such as bathing, let alone the extra cost [4].

Recently, wireless signal has been exploited to alleviate above issues. Received Signal Strength (RSS) based solutions leverage the changes in the signal strength to sense human activity. Radio Frequency (RF) based solutions, such as Frequency Modulated Continuous Wave (FMCW) radar and Doppler radar, can reflect the unique patterns of activities [5]. The implementation of the RSS or RF based methods usually involves two practical issues: one is how to overcome performance degradation due to multi-path fading; the other one is how to relax the dependence on specialized hardware.

Recent advances in wireless communication technology, especially the physical layer channel information extraction,

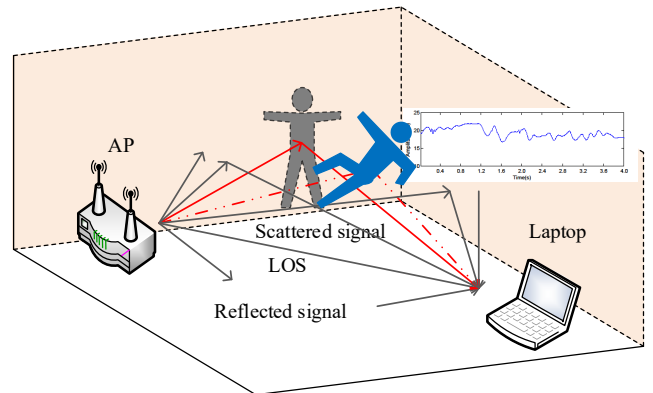

Fig. 1: Human activity affects the WiFi signal propagation.

has provided alternative approach to human activity sensing method using WiFi signals. In particular, the exposed physical layer Channel State Information (CSI) have been used for activity sensing such as falling down [9], smoking [10], breathing beats [11], motion direction inferring [12], [13], hand counting [14]. and hand-free drawing [15]. These systems are based on the observation that CSI is highly sensitive to different human body activities. However, these systems can only recognize a pre-defined set of activities through a supervised learning from observed wireless signals patterns. For more practical cases, these systems do not work as expected. More specifically, an activity might be sensed as abnormal when it appears in the first time, however, it may be considered as normal when more and more instances are observed. Existing abnormal activity sensing methods can not support this, because not all abnormal activity can be predefined, and also the abnormal is a concept that depends on the observation frequency [16].

In this paper, we show the potential to non-invasive abnormal activity sensing by using commodity off-the-shelf (COTS) IEEE 802.11 devices. The key insight is that the time series of CSI values can reflect the unique characteristics of activities in both time and frequency domains. To translate the above ideas into a working system entails several technical challenges: (1) How to extract effective human body features under the subtle signal changes? (2) How to model the abnormal activities, especially for 180 groups of CSI values embedded in one packet extracted from the receiver? (3) The abnormal activities are with different patterns which can not and should not be 
TABLE I: Capabilities of different methods.

\begin{tabular}{cccccccc}
\hline Type & Typical System & Approach & Non-invasive & Device-free & Accurate & Easy for installation & Comprehensive \\
\hline Computer Vision & Xbox Kinect [2] & Camera & $\times$ & $\times$ & $\sqrt{ }$ & $\times$ & $\sqrt{ }$ \\
\hline \multirow{2}{*}{ Wearable Sensor } & BodyScope [4] & Acoustic & $\times$ & $\times$ & $\sqrt{ }$ & $\times$ & $\times$ \\
& TEXIVE [6] & Motion & $\sqrt{ }$ & $\times$ & $\sqrt{ }$ & $\times$ & $\times$ \\
\hline \multirow{2}{*}{ Wireless Signal } & WiTrack [7] & RF & $\sqrt{ }$ & $\times$ & $\sqrt{ }$ & $\times$ & $\times$ \\
& WiGest [8] & RSS & $\sqrt{ }$ & $\sqrt{ }$ & $\times$ & $\sqrt{ }$ & $\times$ \\
& RT-Fall [9] & CSI & $\sqrt{ }$ & $\sqrt{ }$ & $\sqrt{ }$ & $\times$ \\
\hline Wireless Signal & WarnFi & CSI & $\boldsymbol{V}$ & $\boldsymbol{V}$ & $\boldsymbol{V}$ & $\boldsymbol{V}$ & $\boldsymbol{\checkmark}$ \\
\hline
\end{tabular}

simply pre-defined, but how to automatically cluster the human body activities especially in large-scale datasets?

We addressed the above challenges and demonstrated the feasibility of WiFi-based abnormal activity sensing system called WarnFi (Warn of WiFi). As shown in Figure 1, WarnFi consists of a receiver such as a laptop, and a transmitter such as a router. After clustering the wireless signal patterns using non-parametric model, WarnFi senses the abnormal activities under both line-of-sight (LOS) and non-line-of-sight (NLOS) situations. Unlike cameras, WarnFi does not require lighting and works in dark just as well as in light. It requires no dedicated sensor, nor the supervised learning of WiFi signals for pre-defined activities. This unsupervised framework will save the human interventions needed.

Experiment results in different scenarios including apartment, meeting room and bathroom demonstrate that WarnFi can achieve great performance. More specifically, WarnFi accurately senses abnormal activity with an average accuracy of $88.6 \%$, and the accuracy in LOS situations reaches $90.2 \%$ in which the WiFi device can hear multiple APs. We highlight our main contributions as follows

- We propose and validate the feasibility of Channel State Information (CSI) based abnormal activity sensing method.

- We present a CSI grouping algorithm to reduce the overall computation cost, and utilize a density-based mean-shift clustering method to automatically cluster the human body activities.

- We implement WarnFi using commercial WiFi device and extensive evaluations in different scenarios show that WarnFi is robust to the change of environment.

The rest of this paper is organized as follows. Section II discusses the related work. In Section III we present the system design. Section IV presents the design details of WarnFi. We report the empirical evaluation in Section V. Finally, we conclude our paper in Section VI.

\section{RELATED WORK}

\section{A. Computer Vision based Activity Sensing}

Vision based systems use camera to estimate a sequence of abnormal activity [2], [17]. Zhu et al. combined skeleton joint and spatiotemporal features to achieve 3D activity recognition [18]. Chen et al. improved the activity recognition accuracy by combining inertial body sensor and depth camera [19]. However, the sensitivity to lighting conditions and the introduced privacy disclosure remain practical concerns. Besides, for the blind areas such as the bathroom and stairwells, mounting the camera is inconvenient or even illegal.

\section{B. Wearable Sensor based Activity Sensing}

TAHAR [20] achieved a real-time activity classification with a data collection of inertial sensors such as gyroscopes and accelerometers. TEXIVE [6] leverages smartphone sensors to sense texting operations and driving simultaneously. BodyScope [4] records the sounds to classify activities, such as speaking and laughing. However, additional sensors need to be installed or worn, which is difficult for many people to comply with.

\section{Wireless Signal based Activity Sensing}

According to the source of wireless signals, it can be classified in three types: Radio Frequency $(R F)$ based Sensing Systems, RSS based Sensing Systems, and CSI based Sensing Systems. RF based methods require specialized high cost devices. RSS based methods are not enough accurate to provide fine-grained sensing. Channel State Information (CSI) discriminates multi-path characteristics. WiFinger [14] shows the potential to detect and recognize finger gestures available on COTS devices. WiDance [13] leverages Channel State Information (CSI) to extract activity-induced Doppler shifts for inferring motion direction. WifiU [21] extracts the spectrogram signatures to describe the detailed gait patterns for person identification. WiKey [22] exploits the micro-movements of fingers while typing to extract the individual keystrokes. PreFi [23] leverages CSI to analyze customer's product preference. However, they need the supervised learning of WiFi signals for pre-defined activities.

Table I shows the capabilities of different methods. No existing system simultaneously satisfies non-invasive, devicefree, accurate, easy for installation, and comprehensive. The abnormal activity should be dynamic, rather than static so that a frequently observe activity can not be abnormal. More specifically, an activity might be sensed as abnormal when it appears in the first time, however, it may be considered as normal when more and more instances are observed. Existing abnormal activity sensing methods can not support this, because not all abnormal activity can be pre-defined, and also the abnormal is a concept that dependents on the observation frequency. 


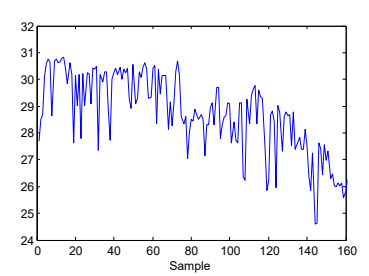

(a) Original CSI

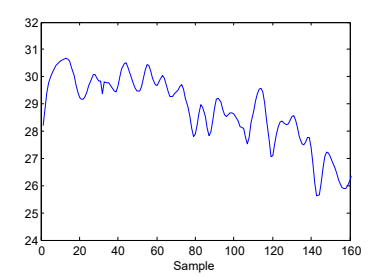

(b) Denoised CSI
Fig. 2: CSI variance of a single subcarrier.

\section{SYSTEM DESIGN}

\section{A. Channel State Information}

In a wireless communication system, channel state information (CSI) portrays how the wireless signal propagates between transmit-receiver antenna pairs. For each subcarrier, CSI exposes the wireless fading phenomenon in the form of amplitude and phase. The channel matrix $\mathbf{H}$ for each subcarrier is modeled as

$$
\mathbf{H}_{i}=\left|\mathbf{H}_{i}\right| e^{j \sin \left(\angle \mathbf{H}_{i}\right)},
$$

in which $\left|\mathbf{H}_{i}\right|$ is the amplitude, and $\angle \mathbf{H}_{i}$ is the phase information. The received signal $\mathbf{R}(t)$ at time $t$ can be expressed as

$$
\mathbf{R}(t)=\mathbf{H}(t) \mathbf{T}(t)+\mathbf{N}(t),
$$

where $\mathbf{T}(t)$ is transmitted vector and $\mathbf{N}(t)$ is the noise vector. For each transmit-receiver antenna pair, we can collect channel state information (CSI) for 30 OFDM subcarriers from the driver of Intel $5300 \mathrm{WiFi}$ NIC as described in [24]. Figure 2 shows the original and denoised CSI.

\section{B. System Overview}

WarnFi is a WiFi-based non-invasive abnormal activity sensing system on COTS WiFi devices. Figure 3 gives the framework of WarnFi, which can be divided into 3 parts: CSI sampling, abnormal activity sensing, and alerting part.

\section{1) CSI Sampling}

The wireless signal continuously propagates between transmit-receiver antenna pairs. The time-series CSI data is sampled from COTS WiFi devices and processed on the computer. Then filtering method is adopted to smooth out noisy data for improving the robustness of environmental change.

2) Abnormal Activity Sensing

WarnFi reduces the overall computation cost and distinguishes those abnormal activities by leveraging data calibration. We employ a non-parametric clustering model and weighted hierarchical majority voting method to automatically cluster the human body activities for abnormal sensing.

\section{3) Alerting}

Based on the clustering model, the next component of our design is decision making, which identifies the "rare and different" activities such as falling down, slipping to the ground, breath pausing, etc.

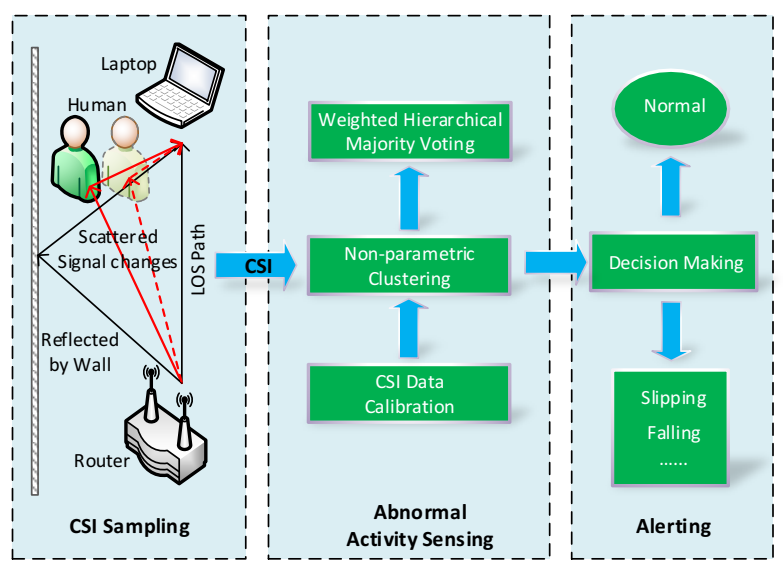

Fig. 3: Framework of WarnFi.

\section{Abnormal Activity Sensing}

WarnFi leverages physical layer information CSI to sense the abnormal activities. It consists of three main components, including CSI data calibration, non-parametric clustering and weighted hierarchical majority voting.

\section{A. Data Calibration}

The goal of data calibration is to avoid the overall computation for decision making and distinguish those abnormal activities. Specifically, our data calibration strategy involves three steps: CSI grouping, feature extraction, feature normalization.

\section{- CSI Grouping}

CSI grouping process groups the whole CSI values into $n$ groups, and the group $i$ is formed by the CSI values at sampling $i, n+i, 2 n+i, \cdots$ etc. It can reduce the overall computation cost.

\section{- Feature Extraction}

In each group, we collect CSI features and transfer them into a uniform format for characterizing the activity. Principal Component Analysis (PCA) is adopted to discover the correlations between CSI streams. Eq. (1) is the CSI feature matrix $\mathbf{F}_{i, j}$ for $n$ groups and 30 subcarriers.

$$
\mathbf{F}_{i, j}=\left[\begin{array}{ccccc}
f_{1,1} & f_{1,2} & f_{1,3} & \cdots & f_{1,30} \\
f_{2,1} & f_{2,2} & f_{2,3} & \cdots & f_{2,30} \\
\vdots & \vdots & \vdots & \ddots & \vdots \\
f_{n, 1} & f_{n, 2} & f_{n, 3} & \cdots & f_{n, 30}
\end{array}\right],
$$

where $f_{i, j}$ is the CSI feature value for the $i^{t h}$ group of the $j^{t h}$ subcarrier.

\section{- Feature Normalization}

As the collected data have different scales, the matrix $\mathbf{F}_{i, j}$ is normalized into the range of 0.0 and 1.0. 


\section{B. Non-parametric Clustering}

We utilize a density-based mean-shift clustering method that requires no supervised information as input parameters. The bandwidth of searching window, which does not need to be pre-defined, is calculated by:

$$
l_{i}=\left\|x_{i}-x_{i, n / 2}\right\|_{1},
$$

where $x_{i, n / 2}$ is the $m / 2$ nearest neighbor of CSI feature $x_{i}(i=1, \cdots, m)$. The clustering method is conducted in the following steps:

1) The new center of the CSI feature in the window is calculated by:

$$
\tilde{x}_{i}=\frac{\sum_{j=1}^{m} x_{i, j} g\left(\left\|\frac{x_{i}-x_{i, j}}{l_{i}}\right\|^{2}\right)}{\sum_{j=1}^{m} g\left(\left\|\frac{x_{i}-x_{i, j}}{l_{i}}\right\|^{2}\right)},
$$

in which $x_{i, j}$ is a CSI feature in the $x_{i}$ search window and $g(x)=q^{\prime}(x)$, in which $q(x)(0 \leqslant x \leqslant 1)$ is the kernel for estimating the density of CSI feature $x_{i}$.

2) Starting from $\tilde{x}_{i}$, step 1 is repeated until a convergence.

\section{Weighted Hierarchical Majority Voting}

Based on the above non-parametric clustering, the goal of our design is to sense the abnormal CSI features in each group.

1) In the first level, a CSI feature is labeled with $M a$ (Majority) if $p_{1} \geq \xi_{1}$, where $p_{1}$ is the probability $x_{i}$ belongs to the majority of the group and $\xi_{1}$ is a threshold. A CSI feature is labeled with $M i$ (Minority) if $p_{1}<\xi_{1}$.

2) In the second level, only the CSI features labeled with $M a$ are selected to vote. $x_{i}$ is labeled with $N$ (Normal) if $p_{2} \geq \xi_{2}$, where $p_{2}$ is the probability $x_{i}$ belongs to the majority of $M a$ in first level; otherwise the CSI feature will be labeled with $A n$ (Ábnormal).

The probability that the CSI feature is regarded as abnormal is $p=1-p_{1} \cdot p_{2}$. The probability of a normal CSI feature being regarded as $M a$ is given by:

$$
P_{N \rightarrow M a}=p_{1} p_{2} \sum_{f=0}^{\left\lceil\frac{n}{2}-1\right\rceil} C_{n}^{f}\left(p_{1} p_{2}\right)^{n-f}\left(1-p_{1} p_{2}\right)^{f},
$$

in which $f$ is the number of abnormal features. The probability of a normal CSI feature being regarded as $M_{i}$ is given similarly:

$$
P_{N \rightarrow M i}=p_{1} p_{2}-P_{N \rightarrow M a} .
$$

The probability of a normal CSI feature being correctly labeled is in Eq. (2).

Similarly, we can derive the probability for an abnormal CSI feature being correctly labeled $P_{A n \rightarrow A n}$. The sensing accuracy of weighted hierarchical majority voting method is calculated by $P_{N \rightarrow N}+P_{A n \rightarrow A n}$, indicating the probability of an activity being correctly classified. Specifically, an activity might be sensed as abnormal when it appears in the first time, however, it may be considered as normal when more and more instances are observed.

$$
\begin{aligned}
& P_{N \rightarrow N}=p_{1} p_{2} \sum_{m=1}^{n} C_{n}^{m}\left(\sum_{a=0}^{\left\lceil\frac{m}{2}-1\right\rceil} C_{m}^{a} P_{N \rightarrow M a}^{m-a} P_{A n \rightarrow M a}^{a}\right) \\
& \sum_{b=0}^{n-m} C_{n-m}^{b} P_{N \rightarrow M i}^{b} P_{A n \rightarrow M i}^{n-m-b}+P_{N \rightarrow M a} \sum_{c=0}^{n} C_{n}^{c} P_{N \rightarrow M i}^{c} P_{A n \rightarrow M i}^{n-c}
\end{aligned} .
$$

\section{EMPIRICAL EVALUATION}

\section{A. Device and Network}

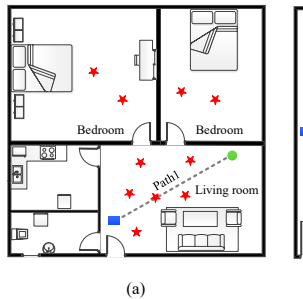

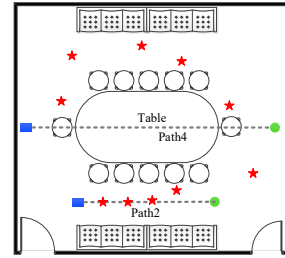

(b)

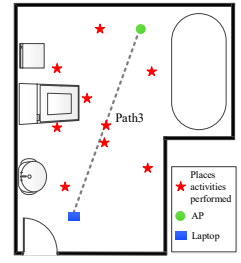

(c)
Fig. 4: Apartment (a), Meeting Room (b), Bathroom (c).

Experiments are conducted on one Think-pad X200 laptop with IEEE 802.11n WiFi network device and a router TPLINK TL-WDR4300. Laptops equipped with Intel 5300 network interface card (NIC) are to establish orthogonal links. The router which is set as access point (AP) runs on $5 \mathrm{GHz}$, while the laptop runs Ubuntu 10.04 which is set as receiver. We chose the $5 \mathrm{GHz}$ band because its wavelength is shorter and it gives better resolutions than $2.4 \mathrm{GHz}$ band. We extract the CSI values of 30 Orthogonal Frequency Division Multiplexing (OFDM) subcarriers from IEEE 802.11 data frames using a modified driver as described in [24]. The pair of WiFi devices communicate with $100 \mathrm{pkts} / \mathrm{s}$. We use MATLAB to analyze the CSI data.

\section{B. Experimental Methodology}

Our primary focus in WarnFi is smart spaces abnormal activity sensing, we then choose three different indoor environments for our evaluation as shown in Figure 4: (a) an apartment covering about $9 \times 9 \mathrm{~m}^{2}$ area with two bedrooms and a living room. (b) a meeting room covering an area of around $9 \times 10$ $\mathrm{m}^{2}$ with a table, dozens of chairs and two groups of sofas; (c) a

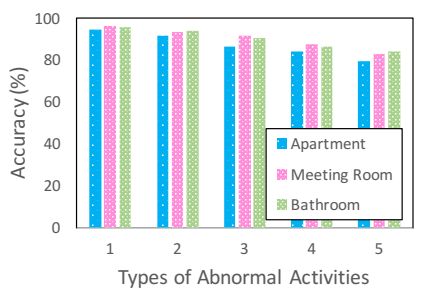

(a) LOS

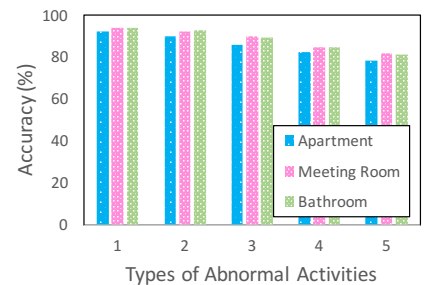

(b) NLOS
Fig. 5: Abnormal activity sensing accuracy in different scenarios. 
TABLE II: Top abnormal activities sensed by WarnFi.

\begin{tabular}{ccc}
\hline & Abnormal Activity & Time \\
\hline 1 & Jump & $03: 05 \mathrm{PM}$ \\
\hline 2 & Running fast & $02: 43 \mathrm{PM}$ \\
\hline 3 & Falling down & $03: 35 \mathrm{PM}$ \\
\hline 4 & Slipping on the ground & $04: 17 \mathrm{PM}$ \\
\hline 5 & Breath pausing & $03: 56 \mathrm{PM}$ \\
\hline
\end{tabular}

bathroom covering about $3 \times 4 \mathrm{~m}^{2}$ area. The length of "Path1", "Path2", "Path3" satisfies that $l 1=l 2=l 3.7$ volunteers are recruited to observe the activities performed by testers via video. The arriving activity will be labeled as normal when most of the volunteers label it as such. If the labeled abnormal activity reoccurs frequently, it will be regarded as normal.

\section{Evaluation Metrics}

1) True Positive Rate (TPR): the proportion of instances that WarnFi senses the abnormal activities correctly among all the abnormal activities.

2) False Positive Rate (FPR): the proportion of instances that WarnFi gives false alarm when actually no abnormal activity exists.

\section{Accuracy of WarnFi}

1) Feasibility of Abnormal Activity Sensing: The accuracy of WarnFi's abnormal activity sensing is evaluated by asking the human to vary activities and location as shown in Figure 4. In Table II. we display the top 5 abnormal activities of WarnFi. The results in Figure 5 show that overall WarnFi in NLOS has an average accuracy of $85.7 \%$ in apartment, $88.6 \%$ in meeting room, and $88.2 \%$ in bathroom environment. This accuracy in LOS can be increased to $87.2 \%$ in apartment, $90.2 \%$ in meeting room, and $90.1 \%$ in bathroom environment. The meeting room and the bathroom environment are in higher accuracy. The reason is that while we adopt PCA to discover the correlations between CSI streams, the environment is still faced with complex electromagnetic interference, especially for apartment. The accuracy indicates that WarnFi is robust in different environments.
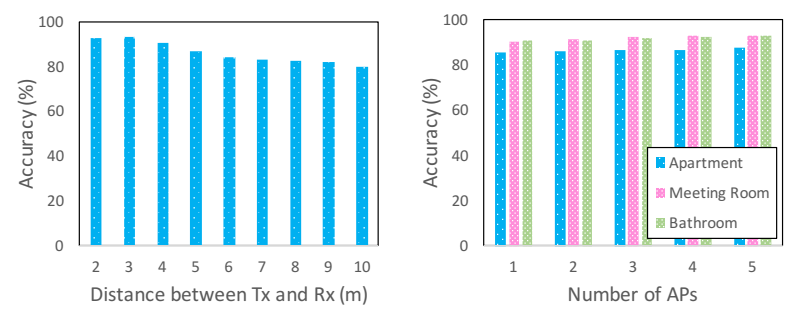

Fig. 6: Impact of different Fig. 7: Impact of different distances between $\mathrm{Tx}$ and APs.

Rx.

The meeting room is chosen to evaluate the performance under different distances between the transmitter which is abbreviated to $T x$ and the receiver which is abbreviated to $R x$. As expected, the sensing error becomes worse when it is more than 5 meters in these scenarios. It is clear that shorter distance leads to higher accuracy, because the received WiFi signals are stronger with shorter communication distances, providing more reliable extraction of CSI to capture the human body movements.

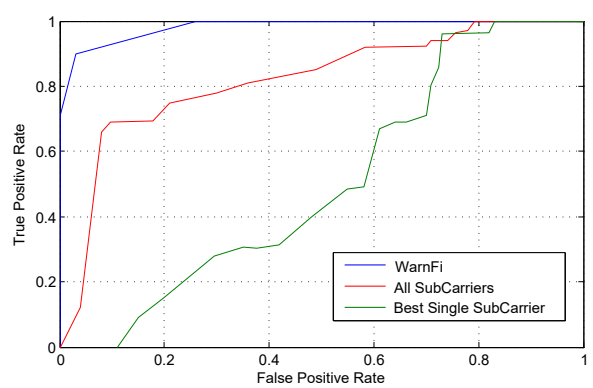

Fig. 8: ROC curves of WarnFi, compared with two baseline methods.

2) Comparision with the Baseline Methods: We use the Receiver Operating Characteristic (ROC) curves to quantitatively evaluate the overall performance of WarnFi against two baseline methods: using all subcarriers without grouping and analysing the best single subcarrier. The CSI values of all subcarriers are directly added up as all subcarriers, and we choose the single subcarrier which has best performance as best single subcarrier. Figure 8 gives ROC curves, which depicts the tradeoff between TPRs and FNRs. The performance for best single subcarrier scheme is worst among them. This is because the wireless signals affected by human activity are scattered in multiple subcarriers, and the fine-grained information can not be easily captured by a single subcarrier.

3) Non-parametric Clustering Accuracy: We compare nonparametric clustering with existing well-known parametric method including k-means, gaussian mixture, DBSCAN [25] and mean-shift clustering. Parametric clustering methods need some predefined parameters. By assuming there are two activity patterns, we set 2 clusters for $k$-means, and gaussian mixture clustering. For DBSCAN, the minimum cluster size is set to 1 . For mean-shift clustering, we set the bandwidth to 0.6 .

Figure 9 demonstrates the clustering accuracy of all these methods. The result demonstrates that when the true number of activity patterns and clusters is the same, all these methods perform well. As the number of abnormal activity types increases, the DBSCAN, mean-shift and non-parametric clustering methods outperform the others slightly.

4) Impact of Different Number of APs: We change the number of transmitter for up to five to illustrate that our measurement accuracy is good enough for abnormal activity sensing. We place the laptop at the center of apartment, meeting room, and bathroom, while APs are placed at the corner of the scenarios. From Figure 7, we have the observation that WarnFi can achieve an average sensing accuracy of $90.2 \%$, 


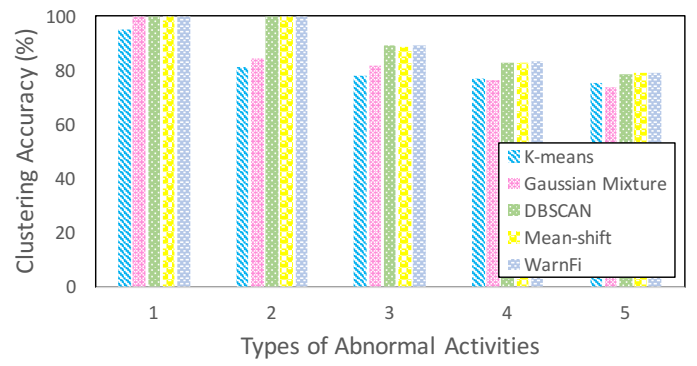

Fig. 9: Comparison of WarnFi with existing clustering methods.

and the accuracy reaches $91.2 \%$ on average when there are more APs. The reason is that there will be more direct path measurements when more APs are included.

\section{CONCLUSION}

In this paper, we propose WarnFi, a non-invasive WiFibased abnormal activity sensing system with only two commodity off-the-shelf (COTS) WiFi devices. First, we present a Channel State Information (CSI) grouping algorithm to reduce the overall computation cost. Then we utilize a densitybased mean-shift clustering method that requires no supervised information as input parameters to sense abnormal activity. Specifically, an activity might be sensed as abnormal when it appears in the first time, however, it may be considered as normal when more and more instances are observed. We compare WarnFi with the baseline methods and extensive experimental results demenstrate that WarnFi has made some progress by proposing an abnormal activity sensing system that simultaneously satisfies the non-invasive, device-free, accurate, easy for installation, and comprehensive.

In addition, we aim to extend this work to achieve that it easily distinguishes the furniture with static target. WarnFi may need a training period to recognize the background of the environment [26]. Besides, WarnFi may separate different humans by tracking them respectively in different dimension [7]. This work will contribute to the development of a comprehensive non-invasive WiFi-based sensing system. Furthermore, building a non-invasive WiFi-based higher accuracy monitoring system is our future work.

\section{ACKNOWLEDGEMENT}

The authors would like to thank our lab volunteers who help us collect the human activity data set. This research is supported by Research of life cycle management and control system for equipment household registration (No. J770011104).

\section{REFERENCES}

[1] V. Chandola, A. Banerjee, and V. Kumar, "Anomaly detection: A survey," ACM computing surveys (CSUR), vol. 41, no. 3, p. 15, 2009.

[2] Microsoft, "X-box kinect," http://www.xbox.com.

[3] O. Oreifej and Z. Liu, "Hon4d: Histogram of oriented 4d normals for activity recognition from depth sequences," in Proceedings of the IEEE Conference on Computer Vision and Pattern Recognition, 2013, pp. 716723.
[4] K. Yatani and K. N. Truong, "Bodyscope: a wearable acoustic sensor for activity recognition," in Proceedings of the 2012 ACM Conference on Ubiquitous Computing. ACM, 2012, pp. 341-350.

[5] G. Wang, Y. Zou, Z. Zhou, K. Wu, and L. M. Ni, "We can hear you with wi-fi!" in Proceedings of the 20th annual international conference on Mobile computing and networking. ACM, 2014, pp. 593-604.

[6] C. Bo, X. Jian, X.-Y. Li, X. Mao, Y. Wang, and F. Li, "You're driving and texting: detecting drivers using personal smart phones by leveraging inertial sensors," in Proceedings of the 19th annual international conference on Mobile computing \& networking. ACM, 2013, pp. 199-202.

[7] F. Adib, Z. Kabelac, D. Katabi, and R. C. Miller, "3d tracking via body radio reflections," in 11th USENIX Symposium on Networked Systems Design and Implementation (NSDI 14), 2014, pp. 317-329.

[8] H. Abdelnasser, M. Youssef, and K. A. Harras, "Wigest: A ubiquitous wifi-based gesture recognition system," in Computer Communications (INFOCOM), 2015 IEEE Conference on. IEEE, 2015, pp. 1472-1480.

[9] H. Wang, D. Zhang, Y. Wang, J. Ma, Y. Wang, and S. Li, "Rt-fall: A real-time and contactless fall detection system with commodity wifi devices," IEEE Transactions on Mobile Computing, 2016.

[10] X. Zheng, J. Wang, L. Shangguan, Z. Zhou, and Y. Liu, "Smokey: Ubiquitous smoking detection with commercial wifi infrastructures," in Computer Communications, IEEE INFOCOM 2016-The 35th Annual IEEE International Conference on. IEEE, 2016, pp. 1-9.

[11] X. Wang, C. Yang, and S. Mao, "Tensorbeat: Tensor decomposition for monitoring multi-person breathing beats with commodity wifi," Acm Transactions on Intelligent Systems Technology, 2017.

[12] H. Wang, H. Wang, H. Wang, H. Wang, and H. Wang, "Widir: walking direction estimation using wireless signals," in ACM International Joint Conference on Pervasive and Ubiquitous Computing, 2016, pp. 351-362.

[13] K. Qian, C. Wu, Z. Zhou, Y. Zheng, Z. Yang, and Y. Liu, "Inferring motion direction using commodity wi-fi for interactive exergames," in CHI Conference, 2017, pp. 1961-1972.

[14] H. Li, W. Yang, J. Wang, Y. Xu, and L. Huang, "Wifinger: talk to your smart devices with finger-grained gesture," in Proceedings of the 2016 ACM International Joint Conference on Pervasive and Ubiquitous Computing. ACM, 2016, pp. 250-261.

[15] L. Sun, S. Sen, D. Koutsonikolas, and K.-H. Kim, "Widraw: Enabling hands-free drawing in the air on commodity wifi devices," in Proceedings of the 21st Annual International Conference on Mobile Computing and Networking. ACM, 2015, pp. 77-89.

[16] J. Yin, Q. Yang, and J. J. Pan, "Sensor-based abnormal human-activity detection," IEEE Transactions on Knowledge Data Engineering, vol. 20, no. 8, pp. 1082-1090, 2008.

[17] "Leap motion," https://www.leapmotion.com

[18] Y. Zhu, W. Chen, and G. Guo, "Fusing spatiotemporal features and joints for $3 \mathrm{~d}$ action recognition," in IEEE Conference on Computer Vision and Pattern Recognition Workshops, 2013, pp. 486-491.

[19] C. Chen, R. Jafari, and N. Kehtarnavaz, "Improving human action recognition using fusion of depth camera and inertial sensors," IEEE Transactions on Human-Machine Systems, vol. 45, no. 1, pp. 51-61, 2015.

[20] J. L. Reyes-Ortiz, L. Oneto, X. Parra, and D. Anguita, "Transition-aware human activity recognition using smartphones," Neurocomputing, vol. 171, no. C, pp. 754-767, 2016.

[21] W. Wang, A. X. Liu, and M. Shahzad, "Gait recognition using wifi signals," in Proceedings of the 2016 ACM International Joint Conference on Pervasive and Ubiquitous Computing. ACM, 2016, pp. 363-373.

[22] K. Ali, A. X. Liu, W. Wang, and M. Shahzad, "Keystroke recognition using wifi signals," in Proceedings of the 21st Annual International Conference on Mobile Computing and Networking. ACM, 2015, pp. 90-102.

[23] N. Pang, D. Zhu, K. Xue, W. Rong, Y. Liu, and C. Ou, "Analyzing customers product preference using wireless signals," 2017.

[24] D. Halperin, W. Hu, A. Sheth, and D. Wetherall, "Tool release: gathering $802.11 \mathrm{n}$ traces with channel state information," ACM SIGCOMM Computer Communication Review, vol. 41, no. 1, pp. 53-53, 2011.

[25] H.-P. Kriegel, P. Kröger, J. Sander, and A. Zimek, "Density-based clustering," Wiley Interdisciplinary Reviews: Data Mining and Knowledge Discovery, vol. 1, no. 3, pp. 231-240, 2011.

[26] M. Zhao, F. Adib, and D. Katabi, "Emotion recognition using wireless signals," in Proceedings of the 22nd Annual International Conference on Mobile Computing and Networking. ACM, 2016, pp. 95-108. 